\title{
One Health as a catalyst for sustainable development
}

To the Editor - The COVID-19 pandemic has highlighted the potential of One Health approaches to accelerate progress towards the sustainable development goals (SDGs), with a deadline of 2030. Recognizing that "the health of humans, domestic and wild animals, plants and ecosystems are closely linked and inter-dependent" ${ }^{\text {, One }}$ Health approaches aim to improve disease management by bringing together medical, veterinary and environmental scientists and policy specialists.

The number of citations to scientific papers reporting One Health research has grown exponentially since the early 2000 s (Fig. 1), and particularly since the One World, One Health conference that was held in New York in 2004. Linking the emergence of zoonotic diseases to environmental degradation, the conference laid out an inclusive approach to preventing epidemic and epizootic disease while maintaining the integrity of ecosystems ${ }^{2}$. One World, One Health formulated the Manhattan principles, calling for "an interdisciplinary approach for combating threats to the health of life on Earth". In the context of the United Nations 2030 Agenda for Sustainable Development, One Health reinforces the ambitions of the SDGs - to anchor health in development, recognizing that good health depends on and contributes to other development goals, underpinning social justice, economic prosperity and environmental protection ${ }^{3}$.

In my opinion, four key challenges need to be tackled for One Health approaches to be successful in their own right, and in the wider context of sustainable development.

The first challenge is to resolve the paradox of unconnected systems. The temporal and spatial dynamics of any system depend on the characteristics of its component parts and on the form of the interactions between them. One Health recognizes this for animals, people and the environment, but the same idea has separately motivated 'Health in All Policies' (Fig. 1) and 'whole of society' approaches, which argue that health should be represented in policies developed across all sectors of government and civil society ${ }^{3,4}$. It has been invoked, too, by complexity and network theories, planetary health, and the view of pandemics as syndemics, where the concurrent spread of multiple infections, such as COVID-19 and HIV/AIDS, is

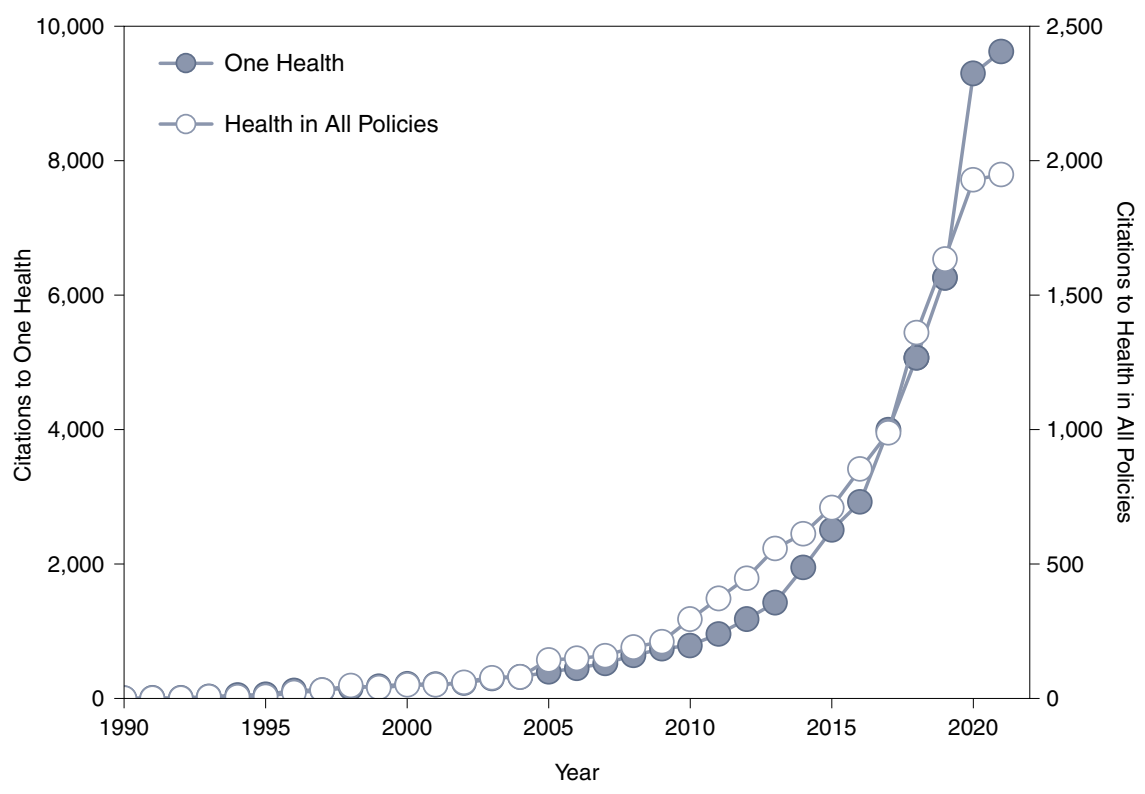

Fig. 1 | Growth in annual citations to journal articles on the topics of One Health and Health in All Policies for the date range 1990-2020. The growth in the number of citations for both topics is exponential, with almost the same growth rate. However, the number of citations to articles on 'One Health' have consistently remained 4-5 times greater than the number for 'Health in All Policies' (including 'Health in All'). Data source: Web of Science (http://www.webofscience.com/).

exacerbated by prevailing social, economic and environmental conditions. The mother of all systems is the network of $17 \mathrm{SDGs}^{3}$. Yet, within the principal health goal (SDG 3), there remains a disconnect between the patient-focused preoccupation of clinical medicine and the population-level objectives of the SDGs. This assortment of overlapping but disjointed frameworks is a signal that, while systemic thinking has broad appeal, systemic action trails far behind. Transdisciplinary research and intersectoral practice are still the exceptions rather than the rule.

This lack of connectedness is one reason why progress towards meeting the SDGs by 2030 is not on track in most regions of the world ${ }^{5}$. Another reason, according to some critics of the sustainable development agenda, is that inclusivity is a recipe for inefficiency ${ }^{6}$. They argue that the SDGs comprise far too many poorly defined targets, with ambiguous criteria for success. They prefer the precision targeting of the millennium development goals (MDGs), predecessors of the SDGs, setting clear priorities in a world of limited resources. They point to the successes of the MDG era - for instance, the number of people living in extreme poverty; the malaria, tuberculosis and under-five mortality rates; and the maternal mortality ratio all fell by about one-half or more between 1990 and 2015 (ref. $^{3}$ ).

This critique of the SDGs sets up the second challenge: to define One Health programmes that are neither too narrow (missing chances for collaboration) nor too broad (difficult to manage and diluting priorities). Rabies is a good test of this challenge ${ }^{7}$. Vaccines that can be given both to animals and people should be effective in controlling zoonotic diseases. The global strategic plan to end human deaths from dog-mediated rabies by 2030 (ref. ${ }^{8}$ ) urges "implementation of the One Health approach, embedded within strong human and animal health services". But what is the optimal design of a One Health programme for rabies control? In general, rabies control programmes now recognize the value of a two-pronged attack on the disease, 
in humans and animals - post-exposure prophylaxis (rabies immunoglobulin and vaccination for people bitten by suspected rabid animals), plus mass vaccination of animal reservoirs, mainly domestic dogs, reduces the risk of human exposure and can potentially eliminate rabies ${ }^{9}$. The details of implementation vary from one setting to another. Success in Vietnam, for instance, was based on the decision to produce dog vaccines domestically (1994) followed by a prime-ministerial directive to prevent and control rabies nationally (1996), later reinforced by an explicitly One Health approach to multisectoral collaboration $(2001)^{10}$.

The third challenge is to align One Health with other approaches to disease control. Containing the spread of antimicrobial resistance (AMR) in farm animals is a case in point. When the antibiotic avoparcin is no longer added to chicken, pig and cattle feed as a prophylactic and growth promoter, the prevalence of resistance to the related antibiotic vancomycin falls in both animals and humans ${ }^{11}$. The One Health message from this and similar studies is clear - use antimicrobials principally for therapy in animals and people, rarely for prophylaxis, and never for growth promotion. Also, cut antimicrobial contamination of the environment ${ }^{12}$. Some take an even broader view, pointing out that reduced meat consumption means fewer farm animals, and a lower demand for antimicrobials in the first place.

But the rational use of antimicrobials on farms and in clinics, consistent with One Health, must be reconciled with other ways of framing the problem of AMR, for example in terms of biosecurity. One Health emphasizes collective responsibility in addressing the social, economic, environmental and medical determinants of antimicrobial use. In contrast, the biosecurity approach adopted by some high-income countries favours medical intervention over stewardship, and national protection against perceived AMR threats from the Global South. The misplaced sense of danger from the South was epitomized by the inappropriate naming in 2009 of
New Delhi metallo- $\beta$-lactamase-1, one member of a group of enzymes that confer resistance to carbapenem antibiotics that has been found worldwide since the 1990s (ref. ${ }^{13}$ ).

Challenge number four is to design One Health programmes that involve everyone who has a stake in the outcome. Monash University's Revitalising Informal Settlements and their Environments (RISE) programme is a good example that shows how overcoming this challenge can pay off ${ }^{4,14}$. More than a billion people living in informal settlements, mostly in low- and middle-income countries, are exposed to pathogens through unsafe water and sanitation. RISE aims to reduce exposure by installing private toilets and hand basins in homes, limiting the release of contaminated sewage and household greywater into the environment, improving flood mitigation and lowering exposure to pollution through rainwater harvesting and wastewater recycling. The engineering solutions are vital to the programme's success, but so is the fact that it has been designed and built with the input of all stakeholders - local governments, financiers and researchers, and residents through community engagement councils.

The consequences of excluding key participants from decisions about essential services, such as water and sanitation, are clear. As an example, the UK House of Commons Environmental Audit Committee reported in January 2022 that only 14\% of English rivers met 'good ecological status' and no river met 'good chemical status' ${ }^{15}$. The reasons are well-known: runoff from agriculture, including animal waste and nitrogen and phosphorous fertilizers, is the biggest polluter of rivers. Added to this are regular discharges of raw sewage into rivers and at seashores by private water companies, and the plastic pollution of waterways is ubiquitous. One Health solutions would rebalance power towards public interest through tighter regulations to prevent the pollution of rivers and coastal waters, inducements and penalties for polluters, and accurate pollution monitoring.

One Health thinking has been brought to the attention of decision makers by the
COVID-19 pandemic because SARS-CoV-2 probably originated, like most emerging viruses, through contact between animals and people in a shared environment. The interdependencies in ecological systems are now acknowledged at the highest levels in political debate. During 2021, in the spirit of the Manhattan principles, G7 leaders promised to "champion an integrated and systems-based One Health approach across all aspects of pandemic prevention, preparedness, detection and response and work to foster a healthier planet". Holding the G7 to that promise will reinforce the view that One Health really can be a catalyst for sustainable development.

\section{Christopher Dye (iD) \\ Department of Biology, University of Oxford, \\ Oxford, UK. \\ $\bigotimes_{\mathcal{e} \text {-mail: christopher.dye@zoo.ox.ac.uk }}$}

Published online: 24 February 2022

https://doi.org/10.1038/s41564-022-01076-1

\section{References}

1. Tripartite and UNEP support OHHLEP's definition of 'One Health'. World Health Organization (1 December 2021).

2. One World, One Health: Building Interdisciplinary Bridges to

Health in a "Globalized World" (One Health Commission, 2004).

3. Dye, C. Science 359, 1337-1339 (2018).

4. Systems-Based Approaches in Public Health: Where Next? (Academy of Medical Sciences, 2021).

5. Sachs, J. et al. Sustainable Development Report 2021 (Cambridge Univ. Press, 2021).

6. Copenhagen Census (accessed 29 November 2021); www.copenhagenconsensus.com

7. Cleaveland, S. et al. Vet. Rec. 175, 188-193 (2014).

8. United Against Rabies Collaboration: First Annual Progress Report (WHO, FAO, OIE, 2019).

9. Cleaveland, S. et al. Philos. Trans. R. Soc. Lond. B Biol. Sci. https://doi.org/10.1098/rstb.2016.0168 (2017).

10. Nguyen, H. T. T. et al. Rev. Sci. Tech. 38, 199-212 (2019).

11. van den Bogaard, A. E., Bruinsma, N. \& Stobberingh, E. E. J. Antimicrob. Chemother. 46, 146-148 (2000).

12. Marshall, B. M. \& Levy, S. B. Clin. Microbiol. Rev. 24, 718-733 (2011).

13. Overton, K. et al. BMJ Glob. Health 6, e006909 (2021).

14. Leder, K. et al. BMJ Open 11, e042850 (2021).

15. Water Quality in Rivers (UK Parliament, 2022).

\section{Acknowledgements}

This article is based on a presentation made at the 22nd European Molecular Biology Laboratory Science and Society Conference, 'One Health: integrating human, animal and environmental health', held on 3 December 2021.

\section{Competing interests}

The author declares no competing interests. 